\title{
Zero-point magnetic exchange interactions
}

\author{
Juba Bouaziz $\odot,{ }^{1,2, *}$ Julen Ibañez-Azpiroz $\odot{ }^{3}$ Filipe S. M. Guimarães $\odot,{ }^{1}$ and Samir Lounis $\oplus^{1,4, \uparrow}$ \\ ${ }^{1}$ Peter Grünberg Institut and Institute for Advanced Simulation, Forschungszentrum Jülich and JARA, Jülich 52425, Germany \\ ${ }^{2}$ Department of Physics, University of Warwick, Coventry CV4 7AL, United Kingdom \\ ${ }^{3}$ Centro de Física de Materiales, Universidad del País Vasco/Euskal Herriko Unibertsitatea, 20018 Donostia, San Sebastián, Spain \\ ${ }^{4}$ Faculty of Physics, University of Duisburg-Essen, 47053 Duisburg, Germany
}

(Received 11 June 2020; revised 12 November 2020; accepted 13 November 2020; published 10 December 2020)

\begin{abstract}
Quantum fluctuations are ubiquitous in physics. Ranging from conventional examples like the harmonic oscillator to intricate theories on the origin of the universe, they alter virtually all aspects of matter, including superconductivity, phase transitions, and nanoscale processes. As a rule of thumb, the smaller the object, the larger its impact. This poses a serious challenge to modern nanotechnology, which aims at total control via atom-by-atom engineered devices. In magnetic nanostructures, high stability of the magnetic signal is crucial when targeting realistic applications in information technology, e.g., miniaturized bits. Here we show that zeropoint spin fluctuations play an important role in determining the fundamental magnetic exchange interactions that dictate the nature and stability of the magnetic state. Based on the fluctuation-dissipation theorem, we show that quantum fluctuations correctly account for the large overestimation of the interactions as obtained from conventional static first-principles frameworks, filling in an important gap between theory and experiment [Zhou et al., Nat. Phys. 6, 187 (2010); Khajetoorians et al., ibid. 8, 497 (2012)]. Our analysis further reveals that zero-point spin fluctuations tend to promote the noncollinearity and stability of chiral magnetic textures such as skyrmions, a counterintuitive quantum effect that inspires practical guidelines for designing disruptive nanodevices.
\end{abstract}

DOI: 10.1103/PhysRevResearch.2.043357

\section{INTRODUCTION}

Matter is constituted by a collection of ions and a surrounding cloud of electrons. These microscopic entities obey quantum mechanical laws that, in addition to thermal fluctuations, involve intrinsic quantum fluctuations, a direct consequence of Heisenberg's uncertainty principle. The presence of fluctuations can alter the collective behavior of particles, modifying the physical properties of matter at the macroscopic level, such as the Curie temperature of magnets [1]. In addition, quantum fluctuations determine the energy of the system at its lowest level, the so-called zero-point energy that provides an extra contribution absent in the classical world. A notorious signature is the long-known Casimir effect [2], in which an attractive force emerges spontaneously between two metallic planes separated by vacuum. However, zero-point effects can emerge in a variety of contexts, including recently found light superconducting compounds like $\mathrm{LaH}_{10}$, a quantum crystal stabilized by atomic zero-point fluctuations [3], nuclear spin-lattice relaxation of molecular

\footnotetext{
*j.bouaziz@fz-juelich.de

†s.lounis@fz-juelich.de

Published by the American Physical Society under the terms of the Creative Commons Attribution 4.0 International license. Further distribution of this work must maintain attribution to the author(s) and the published article's title, journal citation, and DOI.
}

magnets [4], and even the internal degrees of freedom in electrical circuit components [5].

As realized in early works [1], quantum fluctuations play a particularly relevant role concerning a central property of the electron, namely, its spin. Known as zero-point spin fluctuations (ZPSFs), they represent an essential ingredient of itinerant electron magnetism and affect a wide range of phenomena, including high-temperature [6-9] and iron-based $[10,11]$ superconductivity, quantum phase-transitions at $0 \mathrm{~K}$ [12], and potentially even skyrmion lattices [13]. Zero-point spin fluctuations are also predicted to play a notorious role in elemental bulk transition-metal paramagnets and ferromagnets $[14,15]$ by modifying the effective magnitude of the spin moments and possibly inducing spin anharmonic effects [16], which can alter the magnetic stability of the ground state [17].

Zero-point spin fluctuations become increasingly important as the size of the system is decreased down to a handful of atoms, a regime where quantum effects prevail. Due to the tremendous current appeal of such low-dimensional systems in the context of information technology and storage as miniaturized magnetic bits [18-20], it becomes crucial to understand and control the effect of quantum fluctuations over their magnetic properties. In the ultimate limit of a single magnetic adatom deposited on a nonmagnetic substrate, different measurements display contrasting magnetic trends depending on the probing protocol $[18,19,21-24]$; while ensemble measurements based on x-ray magnetic circular dichroism report the presence of a huge magnetic anisotropy energy (MAE) that protects the magnetic moment against external 
(a)

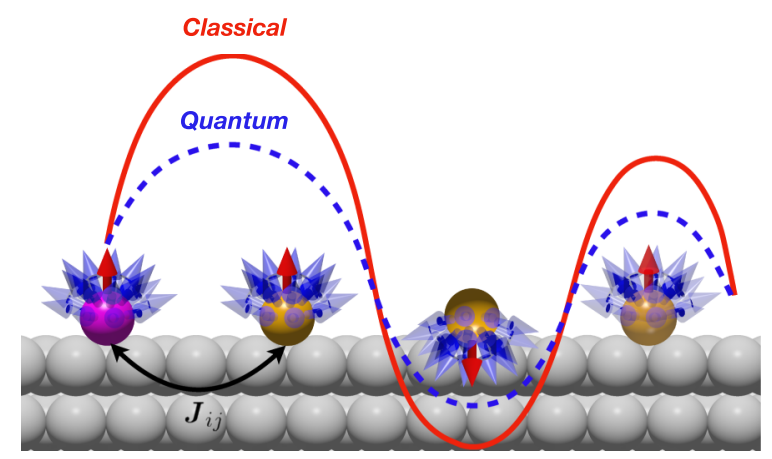

(c)

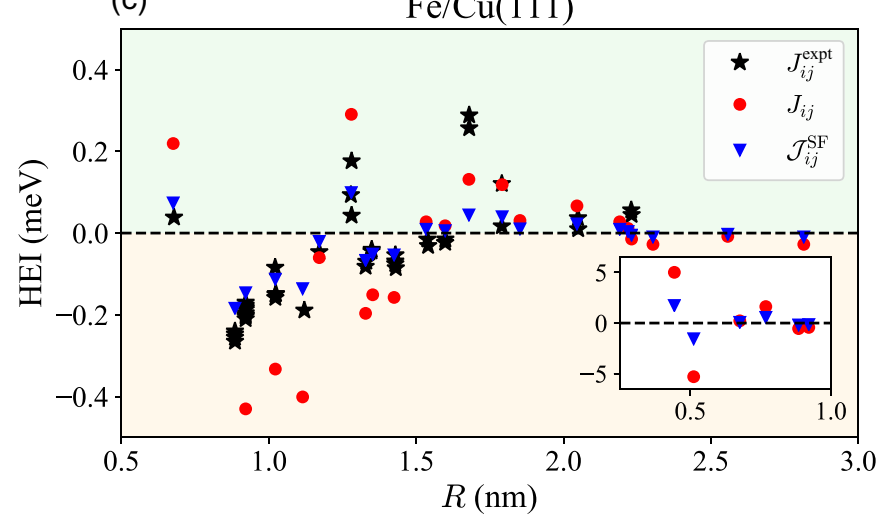

(b)

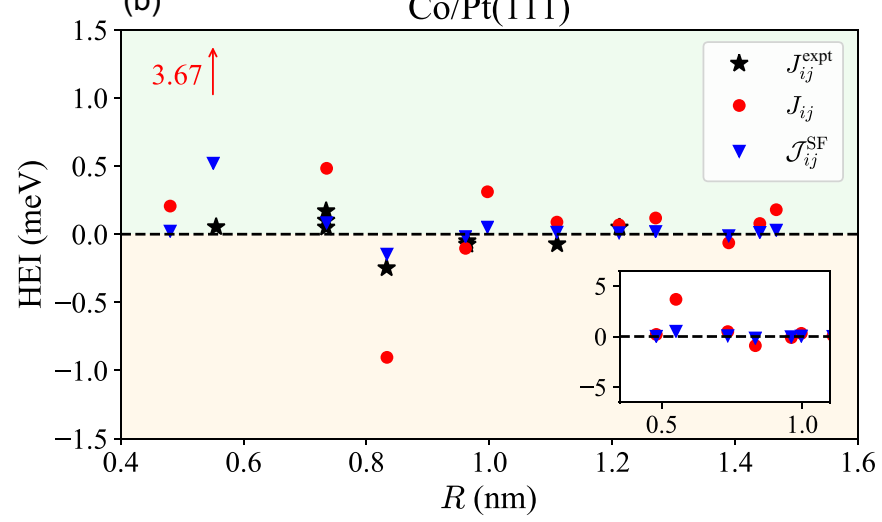

(d)

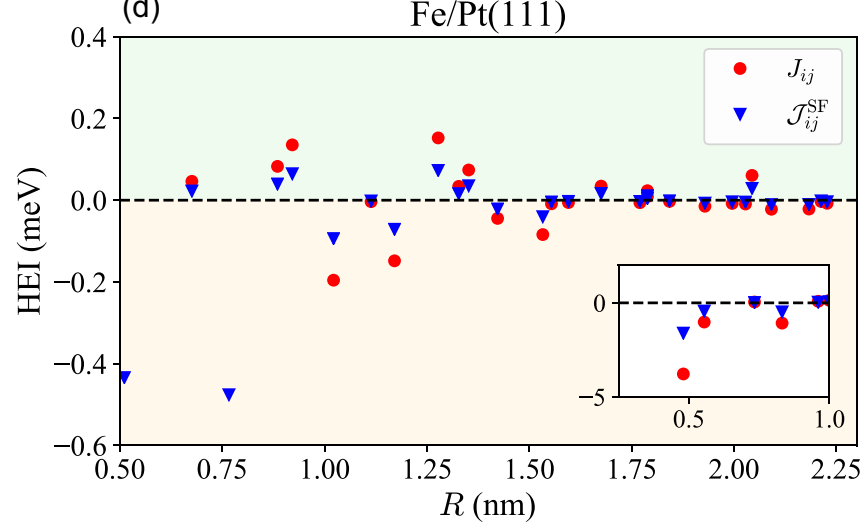

FIG. 1. Distance dependence of the magnetic exchange interaction. (a) Schematic depiction of the magnetic exchange interaction between two fluctuating magnetic moments as a function of interatomic distance (the repeated yellow atom portrays distance dependence). The characteristic RKKY-like dependence is depicted by the oscillating curves, which also exhibit the strong renormalization from the classical (red solid curve) to the quantum (blue dashed curve) prediction. Also shown is the calculated direct exchange interaction as a function of interatomic distance of dimers (b) $\mathrm{Co} / \mathrm{Pt} 111$, (c) Fe/Cu111, and (d) Fe/Pt111. The bare and fluctuation-renormalized Heisenberg exchange interactions are denoted by red circles and blue triangles, respectively. In (b) and (c) the respective experimental data (black stars) from Refs. [27,28] are also included with permission from Nature Publishing Group.

interactions, local probing techniques generally find no stable magnetic signal in the very same systems. To resolve this apparent contradiction, first-principles theory has successfully invoked ZPSF as a mechanism that destroys the magnetic bistability by locally reducing the MAE barrier; the larger the fluctuations, the larger the local reduction, clarifying many of the observed trends $[25,26]$.

Nevertheless, the magnetic signal of multiatomic quantum devices [19,27-31] not only depends on the quantum fluctuations of the single magnetic moments, but also emerges from the way magnetic moments talk to each other via the magnetic exchange interaction (MEI). The impact of local and dynamical correlations on the MEI has been investigated by means of dynamical mean-field theory for elemental bulk and surface materials (see, e.g., Refs. [32,33]) without accounting for spin-orbit coupling. However, the impact of ZPSFs on the different components of the MEI in nanoscale systems formed by a handful of atoms remains to be explored, understood, and eventually quantified theoretically and experimentally. Advances in spin-polarized scanning probing techniques undergone in the past decade allowed pioneering magnetometric measurements of the most fundamental MEI between two magnetic adatoms (i.e., a magnetic dimer) $[19,27,28]$. Remarkably, first-principles calculations based on the local spin-density approximation (LSDA) predict very precisely the nature of the coupling measured experimentally [i.e., ferromagnetic (FM) or antiferromagnetic (AFM)] as a function of the interatomic distance $[27,28]$ [see Fig. 1(a) for a schematic illustration]. However, in direct contrast to this success, the magnitude of the MEI obtained theoretically systematically overestimates the experimental value, with a relative error of more than 100\%, as shown in Figs. 1(b) and 1(c).

In this work we show that quantum fluctuations are behind the gap between standard theory and experiments, providing thereby means to quantify them. In particular, we establish a route based on a first-principles dynamical theory for the realistic evaluation of ZPSFs impacting the MEI. By adapting the coupling constant integral formalism $[1,7,34]$ to the modern framework of time-dependent density functional theory (TDDFT) [35-37], we show that the reduction of the MEI magnitude induced by local and nonlocal ZPSFs results in very good agreement with previous experimental results [27,28]. In addition, our analysis reveals that antisymmetric spin interactions of Dzyaloshinskii-Moriya type are particularly robust against ZPSFs, implying that quantum fluctuations favor the emergence of chiral magnetic textures. These findings highlight the importance of quantum effects in the study of nanoscale magnets and their future applications. 


\section{ZERO-POINT SPIN FLUCTUATIONS FROM FIRST PRINCIPLES}

As the first step in our theoretical analysis, we obtain an expression for the zero-point energy induced by the spin fluctuations, which can then be used to assess the impact on the MEI. For this purpose, we make use of TDDFT linear response theory within the adiabatic LSDA, which represents one of the most powerful tools for analyzing dynamical properties of spins via ab initio methods [35,38]. As a mean-field theory, however, it does not incorporate near-critical fluctuations $[39,40]$, which can be key in low-dimensional systems $[25,26]$. A notable way in which ZPSFs can be incorporated into the theoretical framework is by making use of the socalled fluctuation-dissipation theorem, a fundamental relation that gives access to the magnitude of the local fluctuation of the magnetic moment $[7,25]$

$$
\xi_{i, \pm}^{2}=\frac{1}{\pi} \int_{-\infty}^{+\infty} \operatorname{Im} \chi_{i}^{+-}(\omega) \operatorname{sgn}(\omega) d \omega,
$$

with $\chi_{i}^{+-}(\omega)$ representing spatial average of the interacting transverse susceptibility of a magnetic atom at site $i[36,37]$, evaluated using the Dyson-like equation

$$
\underline{\chi}^{+-}(\omega)=\underline{\chi}_{0}^{+-}(\omega)+\underline{\chi}_{0}^{+-}(\omega) \underline{\mathcal{K}} \underline{\chi}^{+-}(\omega) .
$$

Above, $\underline{\chi}_{0}^{+-}(\omega)$ and $\underline{\mathcal{K}}$ represent the Kohn-Sham susceptibility, describing electron-hole excitations and the exchangecorrelation kernel, respectively; the underline is used to denote the tensorial character of objects. Going one step further, we obtain the zero-point energy associated with the ZPSFs by combining the modern TDDFT framework with the coupling constant integral method [7,34,41], giving rise to the first important relation (see Appendix A for the detailed derivation)

$$
\begin{aligned}
\mathcal{E}_{ \pm}= & -\frac{1}{2 \pi} \operatorname{Im} \operatorname{Tr} \int_{-\infty}^{+\infty}\left\{\underline{\chi}_{0}^{+-}(\omega) \underline{\mathcal{K}}+\ln \left[\underline{1}-\underline{\chi}_{0}^{+-}(\omega) \underline{\mathcal{K}}\right]\right\} \\
& \times \operatorname{sgn}(\omega) d \omega,
\end{aligned}
$$

where the trace runs over the number of magnetic units present in the system.

We now introduce the fluctuation-corrected magnetic exchange interactions $\underline{\mathcal{J}}_{i j}^{\mathrm{SF}}$, which are defined assuming an extended Heisenberg Hamiltonian of the form

$$
\mathcal{E}_{\mathrm{SF}}=-\frac{1}{2} \sum_{i \neq j} \vec{e}_{i} \underline{\mathcal{J}}_{i j}^{\mathrm{SF}} \vec{e}_{j}+\mathcal{E}_{\mathrm{MAE}} .
$$

Here $\mathcal{E}_{\mathrm{SF}}$ represents the fluctuation-corrected band energy of the system and $\mathcal{E}_{\mathrm{MAE}}$ the local contribution from the on-site MAE. In the first-principles framework, $\mathcal{E}_{\mathrm{SF}}$ is given by the sum of the LSDA band energy $\mathcal{E}_{\mathrm{b}}$ and $\mathcal{E}_{ \pm}$of Eq. (3). Then the magnetic exchange interactions renormalized by quantum fluctuations can be obtained from the curvature of $\mathcal{E}_{\mathrm{SF}}$ as

$$
\mathcal{J}_{i j}^{\mathrm{SF}, \alpha \beta} \equiv-\frac{\partial^{2} \mathcal{E}_{\mathrm{SF}}}{\partial e_{i}^{\alpha} \partial e_{j}^{\beta}}=-\frac{\partial^{2} \mathcal{E}_{\mathrm{b}}}{\partial e_{i}^{\alpha} \partial e_{j}^{\beta}}-\frac{\partial^{2} \mathcal{E}_{ \pm}}{\partial e_{i}^{\alpha} \partial e_{j}^{\beta}},
$$

with

$$
J_{i j}^{\alpha \beta} \equiv-\frac{\partial^{2} \mathcal{E}_{\mathrm{b}}}{\partial e_{i}^{\alpha} \partial e_{j}^{\beta}}
$$

the bare exchange interaction parameters that are commonly computed using standard DFT. In Eqs. (5) and (6), $e_{i}^{\alpha}$ denotes the transverse component $\alpha$ of the vector $\vec{e}_{i}$ defining the orientation of the magnetic moment at site $i$.

Therefore, the challenge consists in finding an expression for the extra term $-\frac{\partial^{2} \mathcal{E}_{ \pm}}{\partial e_{i}^{\alpha} \partial e_{j}^{\beta}}$ in Eq. (5). Relying on the magnetic force theorem together with the infinitesimal rotational method [42-44], the correction of the elements of the MEI tensor is determined as (see Appendix C)

$$
\begin{aligned}
-\frac{\partial^{2} \mathcal{E}_{ \pm}}{\partial e_{i}^{\alpha} \partial e_{j}^{\beta}} \simeq & \frac{1}{2 \pi} \operatorname{Im} \operatorname{Tr} \int_{-\infty}^{+\infty} \underline{\chi}^{+-}(\omega) \\
& \times \frac{\partial^{2}\left[\underline{\chi} \underline{\chi}^{+-}(\omega)\right]^{-1}}{\partial e_{i}^{\alpha} \partial e_{j}^{\beta}} \operatorname{sgn}(\omega) d \omega .
\end{aligned}
$$

As a final step, we derive a simple expression for the renormalized MEI in terms of the fluctuating moments by mapping the $a b$ initio $\chi^{+-}(\omega)$ to the one obtained from the Landau-Lifshitz-Gilbert (LLG) model. This allows the identification of the analytical dependence between the dynamical susceptibility and the MEI, giving rise to (see Appendix C)

$$
\mathcal{J}_{i j}^{\mathrm{SF}, \alpha \beta}=J_{i j}^{\alpha \beta}\left[1-\frac{J_{j i}^{\alpha \beta}}{J_{i j}^{\alpha \beta}}\left(\frac{\xi_{i, \pm}^{2}}{M_{i}^{2}}+\frac{\xi_{j, \pm}^{2}}{M_{j}^{2}}+\frac{\xi_{i j, \pm}^{2}}{M_{i} M_{j}}\right)\right],
$$

where $\xi_{i j, \pm}^{2}$ denotes a nonlocal contribution to the ZPSF,

$$
\xi_{i j, \pm}^{2}=\frac{1}{2 \pi} \operatorname{Im} \int_{-\infty}^{+\infty}\left[\chi_{i j}^{+-}(\omega)+\chi_{j i}^{+-}(\omega)\right] \operatorname{sgn}(\omega) d \omega .
$$

Equation (8) represents a central result of the present work, as it provides a quantitative expression showing how the standard tensor of MEI is renormalized by quantum spin fluctuations. As a general and most important trend, this equation shows that fluctuations systematically reduce the predominant symmetric component of the tensor of MEI, given that the hierarchy $\left|\xi_{i, \pm}\right|<\left|M_{i}\right|$ holds in general; we will refer to this important contribution $\left(\mathcal{J}_{i j}^{\alpha \alpha}\right)$ as the Heisenberg exchange interaction (HEI) component. On closer inspection, Eq. (8) further reveals that the antisymmetric piece of the tensor $\left(\mathcal{J}_{i j}^{\mathrm{SF}, \alpha \beta}\right.$ with $\left.\alpha \neq \beta\right)$ giving rise to the Dzyaloshinskii-Moriya interaction (DMI) increases upon the action of ZPSF due to its antisymmetric nature, i.e., the property $J_{i j}^{\alpha \beta}=-J_{j i}^{\alpha \beta}$. The combination of these features may have profound implications for the stability, wavelength, and shape of intensively studied chiral spin textures, such as spirals, skyrmions, and antiskyrmions [45], which are strongly dependent on the ratio of DMI and HEI.

\section{QUANTUM CORRECTIONS AGAINST EXPERIMENTAL EVIDENCE}

With the purpose of connecting the developed firstprinciples framework to the microscopic experimental designs of Refs. [27,28], we investigate magnetic dimers deposited on nonmagnetic metallic substrates as a function of interatomic distance, as schematically depicted in Fig. 1. This analysis exposes the oscillatory behavior of the MEI, bringing into play different exchange mechanisms. In the short-range limit, direct exchange dominates due the strong hybridization among the adatom's $d$ orbitals, while for larger distances the 
(a)

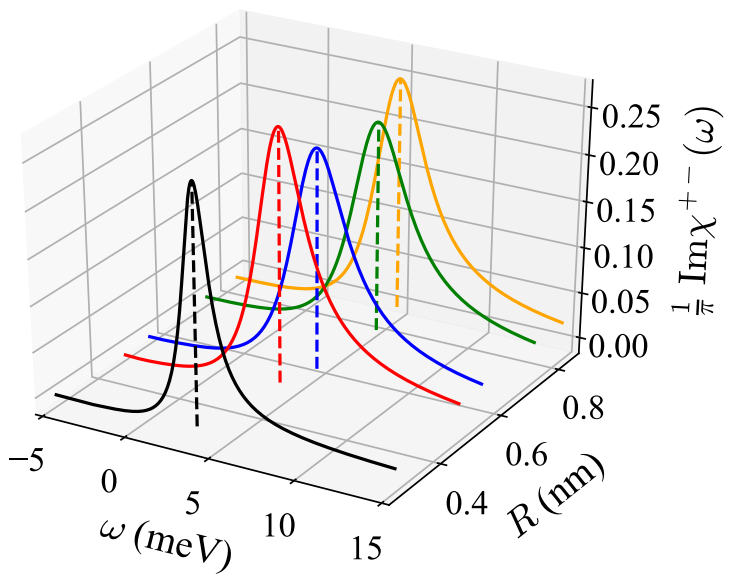

(b)

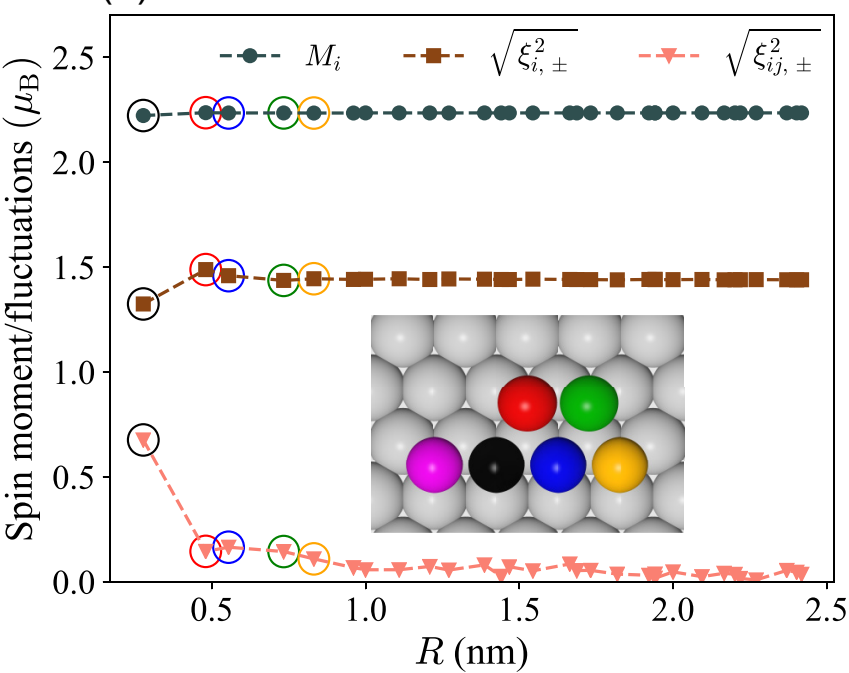

FIG. 2. Spin excitations and zero-point spin fluctuations for Co/Pt(111). (a) Transverse spin-excitation spectra computed at the five shortest interatomic distances. The corresponding atomic configuration is depicted in the inset of (b); the magenta sphere represents a fixed atom, while the other spheres correspond to varying positions of the second atom, whose colors correlate with the lines of $\operatorname{Im} \chi^{+-}(\omega)$. (b) Calculated magnetic moment, local, and nonlocal zero-point spin fluctuations are depicted by gray circles, brown squares, and pink triangles, respectively. Results are shown as a function of interatomic distance; the five shortest distances are encircled by colors that correlate with the atomic configuration portrayed in the inset.

Ruderman-Kittel-Kasuya-Yoshida (RKKY) mechanism prevails, indirectly mediating the interaction via the conduction electrons of the metallic substrate [46-48].

\section{A. Computational details}

We consider $\mathrm{Co}$ and Fe dimers deposited on the fcc stacking sites of the $\operatorname{Pt}(111)$ and $\mathrm{Cu}(111)$ surfaces, respectively; these systems were assessed experimentally, via scanning tunneling microscope (STM) measurements, and theoretically, via bare LSDA calculations, in Refs. [27,28]. In addition, we further investigate an fcc-stacked Fe dimer on $\operatorname{Pt}(111)$ in order to provide theoretical predictions on the fluctuation-renormalized MEI that can be tested in future STM experiments. The dimers are relaxed by $14 \%$ and $20 \%$ for $\mathrm{Cu}(111)$ and $\mathrm{Pt}(111)$ surfaces [49,50]. Briefly, we first determine the nature of the coupling (i.e., FM or AFM) using the infinitesimal rotation method, while the easy axis (MAE) is resolved from the static transverse susceptibility [51]. Subsequently, the spin-excitation spectrum is computed from the corresponding collinear ground-state configuration, in the spirit of the magnetic force theorem approach.

The simulations have been carried out using the scalarrelativistic all-electron Korringa-Kohn-Rostoker Green'sfunction method, including the spin-orbit interaction selfconsistently [52,53]. An angular momentum cutoff of $l_{\max }=$ 3 and a $k$ mesh of $600 \times 600$ have been used for the construction of the Green's functions in real space. The magnetic impurities have been embedded into a real-space impurity cluster containing 56 sites and 30 substrate atoms. The magnetic excitations have been assessed in the framework of TDDFT [35,37], using the adiabatic LSDA for the exchangecorrelation kernel. Finally, the ZPSFs have been obtained from the frequency integral of the imaginary part of the magnetic susceptibility, with a cutoff frequency set at $250 \mathrm{meV}$ (given that the spin-excitation energies are around few meV), after which an $\omega^{-2}$ decay is assumed $[25,26]$.

\section{B. Results}

We begin by briefly describing the calculated groundstate magnetic properties of the adatoms conforming the dimers, which evolve large magnetic moments of $2.3 \mu_{B}$ for $\mathrm{Co} / \mathrm{Pt}(111), 3.3 \mu_{B}$ for $\mathrm{Fe} / \mathrm{Cu}(111)$, and $3.5 \mu_{B}$ for $\mathrm{Fe} / \mathrm{Pt}(111)$. In all the systems, the MAE is of the order of a few meV and favors an out-of-plane orientation. These values are found to be mildly affected by the interatomic distance.

Taking $\mathrm{Co} / \mathrm{Pt}(111)$ as a case study, next we illustrate the main properties of the spin-excitation spectrum of the dimer as a function of interatomic distance in Fig. 2(a). Our calculations reveal that the spectrum is largely dominated by a strong acoustic mode at $\sim 4 \mathrm{meV}$. The optical mode (not shown) is much weaker, lying above $60 \mathrm{meV}$ for the shortest distance and quickly merging with the acoustic mode as the atoms are moved far apart. We note that the position of the acoustic mode correlates with the magnitude of the MAE, while the broadening of the spin excitation is induced by electron-hole excitations $[35,37]$. Our results evidence two clear regimes as a function of interatomic distance: the dimerlike $(\lesssim 0.7 \mathrm{~nm})$ and the atomiclike $(\gtrsim 0.7 \mathrm{~nm})$. In the dimerlike regime, the symmetry is lowered [51] and the interaction between the two magnetic adatoms makes the acoustic mode highly distance dependent, converging to the single-adatom limit (atomiclike regime) as the strength of the interaction decays.

Owing to the relation established by the fluctuationdissipation theorem [cf. Eq. (1)], these two markedly different regimes are translated into the evolution of the local and nonlocal ZPSF depicted in Fig. 2(b); while the size of the dominant local spin fluctuations oscillates between $\sim 1.3 \mu_{B}$ and $\sim 1.5 \mu_{B}$ at short distances, it converges to a steady value 
of $\sim 1.4 \mu_{B}$ in the atomiclike regime. In turn, the nonlocal ZPSFs decay quickly as a function of distance from $\sim 0.7 \mu_{B}$ to virtually zero and are therefore only relevant in the dimerlike regime. Notably, the calculated magnitude of the ZPSF is of the same order as the ground-state magnetic moment itself [also depicted in Fig. 2(b)], a huge relative value for a purely quantum effect.

Having determined the evolution of the spin-excitation spectrum and ZPSFs as a function of interatomic distance (Fig. 2), we now assess the impact of the calculated spin fluctuations on the renormalization of the HEI, namely, the average of the diagonal components of $\mathcal{J}_{i j}^{\mathrm{SF}, \alpha \beta}$ in Eq. (8). Calculated results for $\mathrm{Co} / \mathrm{Pt}(111), \mathrm{Fe} / \mathrm{Cu}(111)$, and $\mathrm{Fe} / \mathrm{Pt}(111)$ are presented in Figs. 1(b), 1(c), and 1(d), respectively; for the two first systems, we additionally include experimental STM data available from Refs. [27,28]. The adatoms couple ferromagnetically (positive HEI) for the nearest-neighbor distance and display a characteristic RKKY decaying oscillatory behavior as the interatomic distance is increased, with different oscillation periods determined by the Fermi surface of the substrate. Notably, the renormalization effects caused by quantum fluctuations fix the disagreement between standard theory and experiments at virtually all interatomic distances, as demonstrated in Figs. 1(b) and 1(c). As revealed by our results, the main role of the fluctuations is to systematically reduce the magnitude of the $\mathrm{HEI}$, correcting for the overestimation arising from the LSDA. We note that in Refs. [27,28], the bare HEI was systematically divided by a factor equal to either 3 or 2 in order to theoretical results closer to experiments. Remarkably, including ZPSFs achieves an excellent comparison without the need of invoking a posteriori parameters, thus proving that quantum fluctuations are a fundamental mechanism at play in these type of low-dimensional magnets.

\section{UNDERSTANDING QUANTUM FLUCTUATIONS VIA THE LLG MODEL}

In order to identify the role of the fundamental factors that determine the behavior of ZPSFs, we resort to the widely used
LLG model for characterizing the microscopic spin dynamics [54]. Our main goal is to obtain an analytical expression of the TDDFT spin susceptibility entering Eq. (7). Then, by working out the evolution of the magnetic moments forming a dimer, we will synthesize the main properties of the local and nonlocal ZPSFs in terms of the physical parameters entering the LLG model.

The LLG equation describing the damped precessional motion of two magnetic moments $\mathbf{M}_{i}(i=1,2)$ on top of a substrate is

$$
\frac{d \mathbf{M}_{i}}{d t}=-\gamma \mathbf{M}_{i} \times \mathbf{B}_{i}^{\mathrm{eff}}+\frac{\eta}{M_{i}} \mathbf{M}_{i} \times \frac{d \mathbf{M}_{i}}{d t},
$$

with $\gamma$ the gyromagnetic ratio and $\eta$ the Gilbert damping. The first term on the right-hand side represents the torque generated by an effective field $\mathbf{B}_{i}^{\text {eff }}=-\partial \mathcal{E} / \partial \mathbf{M}_{i}$, where $\mathcal{E}\left(\left\{\mathbf{M}_{i}\right\}\right)=$ $\sum_{i} E_{i}\left(\mathbf{M}_{i}\right)-\mathbf{M}_{1} \underline{J}_{12} \mathbf{M}_{2} / M^{2}$, with $E_{i}\left(\mathbf{M}_{i}\right)$ denoting the on-site MAE. The second term on the right-hand side of Eq. (10) models the damping process that drives the magnetization back to equilibrium.

The low symmetry of the problem $\left(C_{s}\right)$ dictates that the quantities involved in the LLG equation for a magnetic dimer have tensorial form (see Appendix B). However, for the sake of clarity, in the following we consider a simpler model with $M_{1}=M_{2} \equiv M$ and where the ZPSFs are determined as a function of two central quantities. The first one consists of an effective MEI weighed by the magnetic moment, $J_{\text {eff }}=$ $J_{i j} \gamma / M$. We note that this quantity accounts for the distance dependence of the system, given that a large (small) $J_{i j}$ corresponds to a small (large) interatomic distance. The second ingredient is the Gilbert damping $\eta$, a quantity that is closely connected to the width of the spin-excitation peak [55] shown in Fig. 2(a) and is known to be a key player for local ZPSFs, as it quantifies the magnitude of electron-hole Stoner excitations [25]. In the dimers studied in this work, the calculated values of $\eta$ range between $\sim 0.1$ for $\mathrm{Fe} / \mathrm{Pt}(111)$ and $\sim 0.4$ for Co/Pt(111).

The key information is encoded into the local and nonlocal LLG spin-flip susceptibilities, which can be synthesized as

$$
\chi_{i j}^{+-}(\omega)=\frac{M \gamma}{2} \frac{J_{\text {eff }}+\left[2 K_{\text {eff }}-(1+i \eta) \omega\right] \delta_{i j}}{\left[2 K_{\text {eff }}-(1+i \eta) \omega\right]\left[2 J_{\text {eff }}+2 K_{\text {eff }}-(1+i \eta) \omega\right]},
$$

with $K_{\text {eff }}=\mathcal{M} \gamma / M$, where $\mathcal{M}$ denotes the MAE. The ZPSFs are obtained via the frequency integral of the imaginary part of Eq. (11), which we have numerically computed using the trapezoidal rule in terms of the quantities $J_{\text {eff }}, K_{\text {eff }}$, and $\eta$.

Figure 3 illustrates the LLG solutions for the local and nonlocal contributions to the spin-fluctuation amplitude as a function of $J_{\text {eff }}$ and $\eta$ in the region relevant for experiments (we have set the MAE along the out-of-plane direction, matching the experimental situation). The figure reveals valuable information on the nature of the ZPSFs. First and foremost, quantum fluctuations induce a much larger impact on the AFM regime $\left(J_{\text {eff }}<0\right)$ as compared to the FM one $\left(J_{\text {eff }}>\right.$ $0)$. This result holds for both local and nonlocal contributions and supports the generally assumed notion whereby ferromagnets are more robust against quantum fluctuations than their antiferromagnetic counterparts $[51,56]$. As a second major message, Fig. 3 shows that the magnitudes of both the effective MEI and Gilbert damping play a different role depending on the nature of the magnetic coupling. In the FM regime, $\eta$ tends to significantly increase both local and nonlocal quantum fluctuations (in line with a previous study on the single-adatom case $[25,26]$ ), whereas the effect of $J_{\text {eff }}$ is much milder. In turn, the AFM regime shows a more complex pattern. The effect of $\left|J_{\text {eff }}\right|$ is significant, inducing large local and nonlocal fluctuations as it increases; as for the Gilbert damping, it induces larger local fluctuations but reduces the nonlocal ones, resulting in a competing mechanism. As an important remark we note that for $J_{\text {eff }} \simeq 0$, the nonlocal ZPSFs vanish in both the FM and AFM regions independently of $\eta$, 
(a)

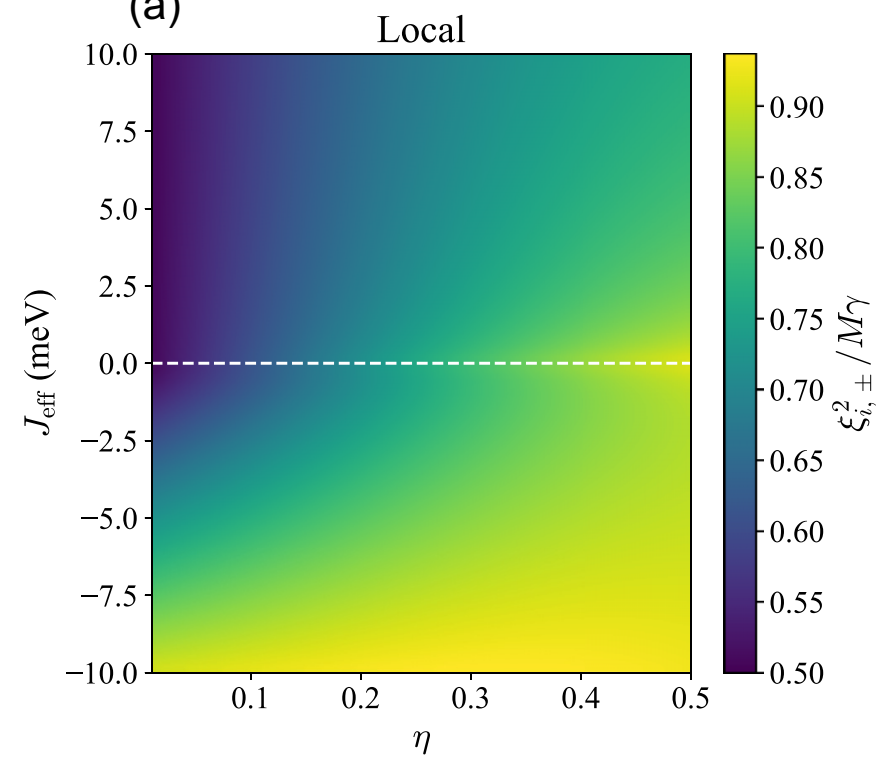

(b)

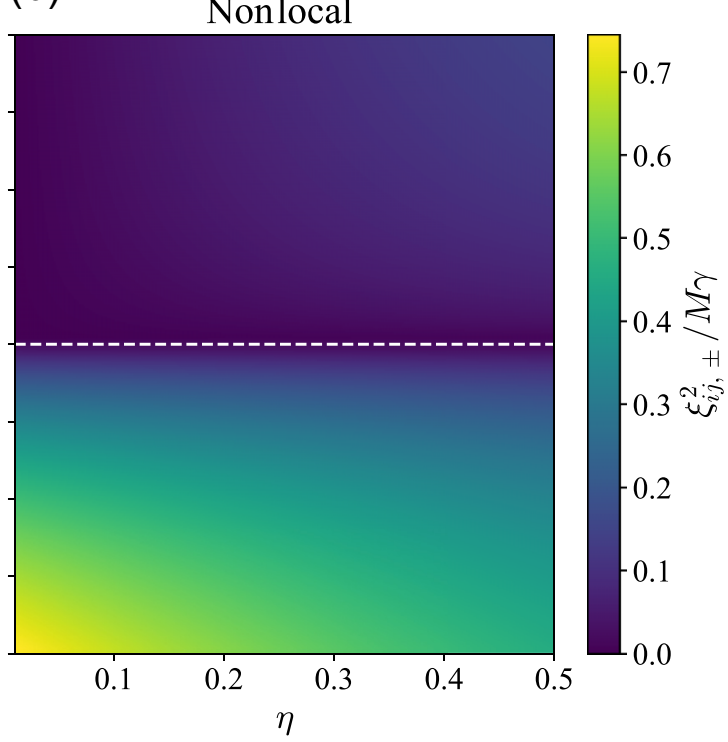

FIG. 3. Colormaps of quantum fluctuations. (a) Local and (b) Nonlocal contributions of the fluctuation-to-magnetization ratio as a function of the effective exchange parameter $J_{\text {eff }}=J_{i j} \gamma / M$ and the Gilbert damping $\eta$. Results were obtained with the Landau-Lifshitz-Gilbert equation of motion with the effective magnetic anisotropy energy $\mathcal{M}, K_{\text {eff }}=\mathcal{M} \gamma / M$, set to $1 \mathrm{meV}$, accounting for an out-of-plane MAE in the order of magnitude of interest.

a feature that explains the quick fall of $\xi_{i j, \pm}^{2}$ observed in the $a b$ initio results of Fig. 2(b).

In order to extract a final lesson, let us focus on the limit of zero damping, i.e., $\eta \rightarrow 0$. This allows the identification of the intrinsic local ZPSFs, which are predominant at large interatomic distances. In this regime, we have $\xi_{ \pm, i}^{2} \simeq \gamma M_{i} / 2$, which is the lower limit of the ZPSFs [see vertical scales in Figs. 3(a) and 3(b)] and provides a similar but inferior approach for the renormalization of the HEI components from Eq. (8):

$$
\mathcal{J}_{i j}^{\mathrm{SF}, \alpha \alpha}=J_{i j}^{\alpha \alpha}\left(1-\frac{\gamma}{2} \frac{M_{i}+M_{j}}{M_{i} M_{j}}\right) .
$$

Notably, this simple expression can be readily incorporated into the conventional DFT framework when computing the MEI with virtually no added cost, given that it only involves ground-state magnetic moments. Note also that Eq. (12) provides a sensible macroscopic limit, whereby the renormalization due to quantum fluctuations tends to vanish for large values of the magnetic moment.

\section{CONCLUSION}

Besides providing insight into the features of the $a b$ initio calculations, the parameter-space map built in Fig. 3 provides a simple recipe to design robust collinear nanomagnets. As its central prediction, an optimal shield against quantum spin fluctuations can be achieved by a combination of large ferromagnetic HEI coupling, relatively low Gilbert damping (i.e., low electronic hybridization), and a strong out-of-plane MAE. On the other hand, in remarkable contrast to the direct-exchange mechanism, ZPSFs play in favor of the antisymmetric nature of the DMI, as proven by Eq. (8) (see also Appendix C). This finding provides an important pre- diction, namely, that quantum spin fluctuations enhance the noncollinearity and the stability of chiral magnetic textures such as skyrmions, governed by the DMI-to-HEI ratio that increases upon the action of ZPSF. This counterintuitive effect appears to be particularly relevant for the ongoing miniaturization process of skyrmions, whose characteristic length scale is approaching the size of the lattice constant and classical predictions start to break down [57].

In summary, we have analyzed the role of quantum spin fluctuations in the fundamental magnetic coupling mechanism of atoms within a TDDFT framework. We have furthermore provided a simple prescription for quantitatively assessing the impact of quantum spin fluctuations in the renormalization of magnetic exchange interactions [cf. Eq. (12)], an expression that can be readily applied to the widespread DFT framework. By applying the approach to magnetic dimers deposited on metallic substrates, we demonstrated that accounting for zeropoint spin fluctuations results in better agreement with the experimentally available data, fixing a relative error of a factor 2-3. Our work therefore suggests that ZPSFs are an important ingredient for the study of nanomagnets and their applications $[29,30]$.

\section{ACKNOWLEDGMENTS}

We are grateful to Jens Wiebe and Roland Wiesendanger for sharing with us the experimental data published in Refs. [27,28]. This work was supported by the European Research Council (ERC) under the European Union's Horizon 2020 research and innovation program (ERC-consolidator Grant No. 681405, DYNASORE). J.I.-A. acknowledges funding from the European Union's Horizon 2020 research and innovation program under the Marie Skłodowska-Curie Grant Agreement No. 839237. We acknowledge the computing time granted by the JARA-HPC Vergabegremium 
and VSR commission on the supercomputer JURECA at Forschungszentrum Jülich [58].

\section{APPENDIX A: TRANSVERSE SPIN FLUCTUATIONS AND ZERO-POINT ENERGY}

In this Appendix we derive the zero-point energy present in the system due to ZPSFs, which emerge as a consequence of the electron-electron interaction. For clarity, a single-band Hubbard model is employed [59], where the Hamiltonian can be written as

$$
\hat{\mathcal{H}}=\hat{\mathcal{H}}^{0}+\hat{\mathcal{H}}^{\mathrm{int}}=\sum_{i j \sigma} t_{i j} c_{i \sigma}^{\dagger} c_{j \sigma}+\sum_{i} U_{i} \hat{n}_{i \uparrow} \hat{n}_{i \downarrow},
$$

where the first term $\hat{\mathcal{H}}^{0}$ accounts for the electrons hopping $t_{i j}$ from site $i$ to $j$ and the second one $\hat{\mathcal{H}}^{\text {int }}$ contains the effective Coulomb electron-electron interaction $U_{i}$. In addition, $\hat{n}_{i \sigma}=c_{i \sigma}^{\dagger} c_{i \sigma}$ is the occupation operator, with $c_{i \sigma}^{\dagger}\left(c_{i \sigma}\right)$ the creation (annihilation) operator of an electron at site $i$ with spin $\sigma$. The sum runs over all the sites of the system. Using the commutation relations between $c_{i \sigma}$ and $c_{i \sigma}^{\dagger}$, the interaction Hamiltonian $\hat{\mathcal{H}}^{\text {int }}$ can be reexpressed in terms of the total occupation number $\hat{n}_{i}=\hat{n}_{i \uparrow}+\hat{n}_{i \downarrow}$ and the net magnetic moment $\hat{m}_{i z}=\hat{n}_{i \uparrow}-\hat{n}_{i \downarrow}$ as [60]

$$
\hat{\mathcal{H}}^{\mathrm{int}}=\frac{1}{4} \sum_{i} U_{i}\left[\hat{n}_{i}^{2}-\left(\hat{m}_{i}^{z}\right)^{2}\right] .
$$

Considering that $\hat{S}_{i z}=\frac{\hat{m}_{i}^{z}}{2}$ and $\hat{S}_{i z}^{2}=\hat{S}_{i}^{2}-\frac{1}{2}\left(\hat{S}_{i+} \hat{S}_{i-}+\hat{S}_{i-} \hat{S}_{i+}\right)$, Eq. (A2) can be recast in terms of spin operators

$$
\mathcal{H}^{\text {int }}=\sum_{i} U_{i}\left(\frac{\hat{n}_{i}^{2}}{4}-\hat{S}_{i}^{2}\right)+\frac{1}{2} \sum_{i} U_{i}\left(\hat{S}_{i+} \hat{S}_{i-}+\hat{S}_{i-} \hat{S}_{i+}\right) .
$$

The resulting interaction energy $\mathcal{E}_{\text {int }}$ can be obtained using the coupling constant integral method [7,34,41]. Using $\lambda$ as a constant parameter that scales $U_{i}$ adiabatically,

$$
\mathcal{E}_{\text {int }}=\sum_{i} U_{i} \int_{0}^{1} d \lambda\left\langle\frac{\hat{n}_{i}^{2}}{4}-\hat{S}_{i}^{2}\right\rangle_{\lambda}+\frac{1}{2} \sum_{i} U_{i} \int_{0}^{1} d \lambda\left\langle\hat{S}_{i+} \hat{S}_{i-}+\hat{S}_{i-} \hat{S}_{i+}\right\rangle_{\lambda} .
$$

The fluctuation energy $\Delta \mathcal{E}$ is defined [7] as the difference between the total $\mathcal{E}_{\text {int }}$ calculated in Eq. (A4) and the Hartree-Fock energy (mean-field energy), denoted here by $\langle\cdots\rangle_{0}$. Therefore,

$$
\begin{aligned}
\Delta \mathcal{E}= & \sum_{i} U_{i} \int_{0}^{1} d \lambda\left(\left\langle\frac{\hat{n}_{i}^{2}}{4}-\hat{S}_{i}^{2}\right\rangle_{\lambda}-\left\langle\frac{\hat{n}_{i}^{2}}{4}-\hat{S}_{i}^{2}\right\rangle_{0}\right) \\
& +\frac{1}{2} \sum_{i} U_{i} \int_{0}^{1} d \lambda\left(\left\langle\hat{S}_{i+} \hat{S}_{i-}+\hat{S}_{i-} \hat{S}_{i+}\right\rangle_{\lambda}-\left\langle\hat{S}_{i+} \hat{S}_{i-}+\hat{S}_{i-} \hat{S}_{i+}\right\rangle_{0}\right),
\end{aligned}
$$

where $\Delta \mathcal{E}$ includes longitudinal and transverse spin fluctuations, as well as charge fluctuations. We focus our subsequent analysis on the transverse spin-fluctuation contribution alone for two main reasons: First, it represents the largest contribution by far for low-dimensional magnets [25], and second, it is the relevant piece for the analysis of the magnetic stability since its energy scale coincides with that of the magnetic anisotropy energy (meV). Consequently, we focus on the transverse spin-fluctuation energy denoted by $\mathcal{E}_{ \pm}$and given by

$$
\begin{aligned}
\mathcal{E}_{ \pm}= & \frac{1}{2} \sum_{i} U_{i} \int_{0}^{1} d \lambda\left(\left\langle\hat{S}_{i+} \hat{S}_{i-}+\hat{S}_{i-} \hat{S}_{i+}\right\rangle_{\lambda}\right. \\
& \left.-\left\langle\hat{S}_{i+} \hat{S}_{i-}+\hat{S}_{i-} \hat{S}_{i+}\right\rangle_{0}\right) .
\end{aligned}
$$

This equation can be rewritten using the spin-flip susceptibilities $\chi_{i}^{+-}(\omega)$ and $\chi_{i}^{-+}(\omega)$ via the fluctuation-dissipation theorem [61]

$$
\begin{aligned}
\left\langle\hat{S}_{i+} \hat{S}_{i-}\right\rangle+\left\langle\hat{S}_{i-} \hat{S}_{i+}\right\rangle & =\frac{1}{\pi} \int_{0}^{+\infty}\left[\operatorname{Im} \chi_{i}^{+-}(\omega)+\operatorname{Im} \chi_{i}^{-+}(\omega)\right] d \omega \\
& =\frac{1}{\pi} \int_{-\infty}^{+\infty} \operatorname{Im} \chi_{i}^{+-}(\omega) \operatorname{sgn}(\omega) d \omega .
\end{aligned}
$$

The last line has been obtained using the symmetry relation $\left[\chi^{-+}(\omega)\right]^{*}=\chi^{+-}(-\omega)$. The transverse spin-fluctuation contribution to $\mathcal{E}_{ \pm}$[Eq. (A6)] can be recast into [7]

$\mathcal{E}_{ \pm}=\frac{1}{2 \pi} \operatorname{Im} \operatorname{Tr} \int_{0}^{1} d \lambda \int_{-\infty}^{+\infty} \underline{U}\left[\underline{\chi}_{\lambda}^{+-}(\omega)-\underline{\chi}_{0}^{+-}(\omega)\right] \operatorname{sgn}(\omega) d \omega$.

Above, the trace runs over the number of atomic sites present in the system. In addition, $\underline{U}$ is a diagonal matrix containing the elements $U_{i}$ and $\underline{\chi}_{0(\lambda)}^{+-}(\omega)$ is the Hartree-Fock $\left(\lambda U_{i^{-}}\right.$ interacting state) susceptibility. In the random-phase approximation we have $\underline{\chi}_{\lambda}^{+-}(\omega)=\underline{\chi}_{0}^{+-}(\omega)+\underline{\chi}_{0}^{+-}(\omega) \lambda \underline{U}_{\underline{\chi}}^{+-}(\omega)$ and $\mathcal{E}_{ \pm}$finally reads

$$
\begin{aligned}
\mathcal{E}_{ \pm}= & -\frac{1}{2 \pi} \operatorname{Im} \operatorname{Tr} \int_{-\infty}^{+\infty}\left\{\underline{\chi}_{0}^{+-}(\omega) \underline{U}\right. \\
& \left.+\ln \left[\underline{1}-\underline{\chi}_{0}^{+-}(\omega) \underline{U}\right]\right\} \operatorname{sgn}(\omega) d \omega .
\end{aligned}
$$

Notably, this expression can be used in the framework of TDDFT by making the connections [36]

$$
\begin{aligned}
\chi_{0, i j}^{+-}(\omega) & \rightarrow \chi_{\mathrm{KS}, i j}^{+-}(\omega)=\int d \mathbf{r} \int d \mathbf{r}^{\prime} \chi_{\mathrm{KS}, i j}^{+-}\left(\mathbf{r}, \mathbf{r}^{\prime}, \omega\right), \\
U_{i} & \rightarrow \mathcal{K}_{i}=\int d \mathbf{r} \int d \mathbf{r}^{\prime} \mathcal{K}_{i}\left(\mathbf{r}, \mathbf{r}^{\prime}\right),
\end{aligned}
$$


with $\chi_{\mathrm{KS}}^{+-}$the Kohn-Sham susceptibility and $\mathcal{K}_{i}$ the adiabatic exchange-correlation kernel, which is local in space. Note that the radial integrals are performed around each atomic site $i$ or $j$.

\section{APPENDIX B: MAPPING TDDFT TO THE LANDAU-LIFSHITZ-GILBERT EQUATION}

In order to interpret results obtained in the framework of TDDFT calculations, we consider a Heisenberg model for localized magnetic moments, whose dynamics are dictated by the LLG equation [54]

$$
\frac{d \mathbf{M}_{i}}{d t}=-\gamma \mathbf{M}_{i} \times \mathbf{B}_{i, \mathrm{eff}}(t)+\eta \frac{\mathbf{M}_{i}}{M_{i}} \times \frac{d \mathbf{M}_{i}}{d t} .
$$

Above, the first term accounts for the precession of the magnetic moment while the second one takes into account relaxation effects, with $\eta$ defining the Gilbert damping parameter. The effective field $\mathbf{B}_{i \text {, eff }}(t)=\mathbf{B}_{i \text {, ext }}(t)+\mathbf{B}_{i}(t)$ includes the transverse time-dependent external magnetic field driving the magnetization out of its equilibrium orientation $\mathbf{B}_{i, \text { ext }}(t)$ as well as internal contributions $\mathbf{B}_{i}(t)$ originating from the anisotropy and exchange interactions [see Eq. (B3)]. The internal fields are obtained from the magnetic part of the internal energy $\mathcal{E}_{\mathrm{M}}$ (see the main text) of these localized moments

$$
\mathcal{E}_{\mathrm{M}}=-\sum_{i} \mathbf{M}_{i} \cdot \frac{K_{i}}{M_{i}^{2}} \cdot \mathbf{M}_{i}-\frac{1}{2} \sum_{\substack{i j \\ i \neq j}} \mathbf{M}_{i} \cdot \frac{\underline{J}_{i j}}{M_{i} M_{j}} \cdot \mathbf{M}_{j},
$$

where $\mathbf{M}_{i}$ and $\underline{K}_{i}$ are the magnetic moment and the magnetocrystalline anisotropy tensor of site $i$, respectively, and $\underline{J}_{i j}$ is the magnetic exchange interaction tensor between sites $i$ and $j$. The effective magnetic field acting on the magnetic moment at site $i$ is defined as

$$
\begin{aligned}
& \qquad \mathbf{B}_{i, \text { eff }}=-\frac{\partial \mathcal{E}_{\mathrm{M}}}{\partial \mathbf{M}_{i}} \\
& =\frac{2 \underline{K_{i}}}{M_{i}^{2}} \cdot \mathbf{M}_{i}+\sum_{j \neq i} \frac{J_{i j}}{M_{i} M_{j}} \cdot \mathbf{M}_{j} . \\
& \qquad\left(\frac{B_{i}^{z}}{M_{i}}-\lambda_{i i}^{\alpha \alpha}-i \frac{\eta \omega}{\gamma M_{i}}\right) M_{i}^{\alpha}-\sum_{j \neq i} \lambda_{i j}^{\alpha \alpha} M_{j}^{\alpha}+\varepsilon_{\alpha \beta} \\
& \text { where }\{\alpha, \beta\}=\{x, y\} \text { designate the transverse blocks and } \varepsilon_{\alpha \beta} \\
& \text { is the Levi-Cività symbol. Equation }(\mathrm{B} 7) \text { defines the inverse } \\
& \text { of the magnetic susceptibility in terms of spin-dynamics } \\
& \text { parameters. Furthermore, the quantity of interest for the } \\
& \text { evaluation of } \mathcal{E}_{ \pm} \text {is the transverse spin susceptibility in the } \\
& \{+,-\} \text { basis }[\text { see Eq. (A9)], which reads } \\
& \qquad-1(\omega)=-\left(\frac{2 B_{i}^{z}}{M_{i}}-\lambda_{i i}^{x x}+\lambda_{i i}^{y y}\right)+\frac{2 \omega}{\gamma M_{i}}(i \eta-1), \\
& {\left[\chi^{+-}(\omega)\right]_{i j}^{-1}(\omega)=-\left(\lambda_{i j}^{x x}+\lambda_{i j}^{y y}\right)+i\left(\lambda_{i j}^{x y}+\lambda_{i j}^{y x}\right) .}
\end{aligned}
$$$$
\left(\frac{B_{i}^{z}}{M_{i}}-\lambda_{i i}^{\alpha \alpha}-i \frac{\eta \omega}{\gamma M_{i}}\right) M_{i}^{\alpha}-\sum_{j \neq i} \lambda_{i j}^{\alpha \alpha} M_{j}^{\alpha}+\varepsilon_{\alpha \beta}\left(i \frac{\omega}{\gamma M_{i}}-\varepsilon_{\alpha \beta} \lambda_{i i}^{\alpha \beta}\right) M_{i}^{\beta}-\sum_{j \neq i} \lambda_{i j}^{\alpha \beta} M_{j}^{\beta}=B_{i, \mathrm{ext}}^{\alpha},
$$

Next, in order to relate the magnetic susceptibility obtained within linear response TDDFT to the LLG model, Eq. (B1) is solved using linear response theory. Analytical expressions for the magnetic susceptibility of the dimer in arbitrary noncollinear magnetic configurations are obtained. For convenience, the response function is computed in the local frame of the magnetic moments $\mathbf{M}_{i}$. Therefore, both local and nonlocal components of $\mathbf{B}_{i}$ are rotated into the local frame of $\mathbf{M}_{i}$. This gives rise to a magnetic anisotropy tensor $\underline{K}_{i}^{\prime}$ and an exchange tensor $\underline{J}_{i j}^{\prime}$. Starting from a ferromagnetic configuration where all the moments point along the $z$ direction,

$$
\begin{aligned}
\underline{J}_{i j}^{\prime} & =\mathcal{R}_{\alpha}\left(\theta_{i}\right) \underline{J}_{i j} \mathcal{R}_{\beta}^{\mathrm{T}}\left(\theta_{j}\right), \\
\underline{K}_{i}^{\prime} & =\mathcal{R}_{\alpha}\left(\theta_{i}\right) \underline{K}_{i} \mathcal{R}_{\beta}^{\mathrm{T}}\left(\theta_{i}\right),
\end{aligned}
$$

where $\mathcal{R}_{\alpha}(\theta)$ defines a vector rotation of an angle $\theta$ around the $\alpha$ axis. Thus, $\underline{J}_{i j}^{\prime}$ and $\underline{K}_{i}^{\prime}$ are dependent on the magnetic orientations. Furthermore, in the linear response regime the following assumptions apply: vanishing torque at equilibrium $\mathbf{M}_{i} \times$ $\mathbf{B}_{i}=0$ and small deviations from the equilibrium $M_{i}^{z}=M_{i}$ and $M_{i}^{x}, M_{i}^{y} \ll M_{i}^{z}$. Within this approximation, the transverse components of $\mathbf{B}_{i, \text { eff }}$ can be related to the magnetization as

$$
\begin{aligned}
B_{i, \mathrm{eff}}^{\alpha}(t) & =B_{i, \mathrm{ext}}^{\alpha}(t)+\sum_{j \beta} \lambda_{i j}^{\alpha \beta} M_{j}^{\beta}(t), \quad\{\alpha, \beta\}=\{x, y\}, \\
\lambda_{i i}^{\alpha \beta} & =\frac{2 K_{i}^{\alpha \beta}}{M_{i}^{2}}, \quad \lambda_{i j}^{\alpha \beta}=\frac{J_{i j}^{\prime \alpha \beta}}{M_{i} M j},
\end{aligned}
$$

while the longitudinal one is static and reads

$$
B_{i, \mathrm{eff}}^{z}=\sum_{j \neq i} \frac{J_{i j}^{\prime z z}}{M_{i}}+\frac{2 K^{\prime z z}}{M_{i}}
$$

Plugging the generalized form of the effective field [Eq. (B5)] into the frequency-dependent LLG equation [Fourier transform of Eq. (B1)], we obtain
The above result enables the extraction of the spin-dynamics parameters by performing a one-to-one mapping to the dynamical susceptibility obtained from TDDFT calculations with first-principles accuracy [51] and is utilized to determine the zero-point spin-fluctuation correction to exchange interactions (see Appendix C).

\section{APPENDIX C: INFINITESIMAL ROTATIONS}

In the preceding Appendix we provided a connection between the spin-flip magnetic susceptibility and the Heisenberg exchange parameters. We now employ these relations to evaluate the contribution of the fluctuations to the exchange interactions, which leads to parameters that closely match the experimental ones (see the main text). In practice, this is achieved by computing the variation of $\mathcal{E}_{ \pm}$upon infinitesimal rotations of the magnetic moments and proceeding to some approximations. Thus, we first compute the change 
in the Kohn-Sham susceptibility $\underline{\chi}_{\mathrm{KS}}^{+-}(\omega)$ with respect to a modification of the $\alpha$ component of the vector $\mathbf{e}_{i}=\frac{\mathbf{M}_{i}}{M_{i}}$,

$$
\begin{aligned}
\frac{\partial \underline{\chi}_{\mathrm{KS}}^{+-}(\omega)}{\partial e_{i}^{\alpha}}= & \frac{\partial}{\partial e_{i}^{\alpha}}\left\{\left[\underline{\chi}^{+-}(\omega)\right]^{-1}+\underline{\mathcal{K}}\right\}^{-1} \\
= & -\left\{\left[\underline{\chi}^{+-}(\omega)\right]^{-1}+\underline{\mathcal{K}}\right\}^{-1} \frac{\partial\left[\underline{\chi}^{+-}(\omega)\right]^{-1}}{\partial e_{i}^{\alpha}} \\
& \times\left\{\left[\underline{\chi}^{+-}(\omega)\right]^{-1}+\underline{\mathcal{K}}\right\}^{-1} \\
\simeq & -\underline{\mathcal{K}}^{-1} \frac{\partial\left[\underline{\chi}{ }^{+-}(\omega)\right]^{-1}}{\partial e_{i}^{\alpha}} \underline{\mathcal{K}}^{-1},
\end{aligned}
$$

where $\chi^{+-}(\omega)$ is the spatial average of the spin-flip susceptibility computed within TDDFT and $\mathcal{K}$ is assumed to be independent of $e_{i}^{\alpha}$ (i.e., isotropic). Note that the expression (C1) was obtained in the limit of magnetic anisotropy energy $\underline{K}_{i}$ and magnetic exchange interaction $\underline{J}_{i j} \ll \mathcal{K}_{i}$, which permits one to disentangle the collective spin excitations and the Stoner contributions to the ZPSF energy. Indeed, $\mathcal{K}_{i}$ is on the order of $\mathrm{eV}$, while the energy scale of the spin excitations is the range of a few meV, as studied in the present work. In addition, as we checked analytically and numerically, the frequency decay of the spin susceptibility [61] leads to vanishing ZPSF energy contributions at high frequencies. Thus, the variation of the integrand defined in Eq. (A9) due an infinitesimal rotation of $\mathbf{e}_{i}$ is written as

$$
\begin{gathered}
\frac{\partial}{\partial e_{i}^{\alpha}}\left\{\underline{\chi}_{\mathrm{KS}}^{+-}(\omega) \underline{\mathcal{K}}+\ln \left[\underline{1}-\underline{\chi}_{\mathrm{KS}}^{+-}(\omega) \underline{\mathcal{K}}\right]\right\} \\
=\underline{\chi}^{+-}(\omega) \frac{\partial\left[\underline{\chi}^{+-}(\omega)\right]^{-1}}{\partial e_{i}^{\alpha}} .
\end{gathered}
$$

To access the magnetic interactions (i.e., curvature of the energy [42]) a second differentiation with respect to another independent variable $e_{j}^{\beta}$ is performed:

$$
\begin{aligned}
& \frac{\partial^{2}}{\partial e_{i}^{\alpha} \partial e_{j}^{\beta}}\left\{\underline{\chi}_{\mathrm{KS}}^{+-}(\omega) \underline{\mathcal{K}}+\ln \left[\underline{1}-\underline{\chi}_{\mathrm{KS}}^{+-}(\omega) \underline{\mathcal{K}}\right]\right\} \\
& \quad=\left[\frac{\partial \underline{\chi}^{+-}(\omega)}{\partial e_{i}^{\beta}} \frac{\partial\left[\underline{\chi}^{+-}(\omega)\right]^{-1}}{\partial e_{j}^{\alpha}}+\underline{\chi}^{+-}(\omega) \frac{\partial^{2}\left[\underline{\chi}^{+-}(\omega)\right]^{-1}}{\partial e_{i}^{\alpha} \partial e_{j}^{\beta}}\right] .
\end{aligned}
$$

The frequency integral of this equation leads to the ZPSF correction of the exchange interactions:

$$
\frac{\partial^{2} \mathcal{E}_{ \pm}}{\partial e_{i}^{\alpha} \partial e_{j}^{\beta}}=-\frac{1}{2 \pi} \operatorname{Im} \operatorname{Tr} \int_{-\infty}^{+\infty}\left[\frac{\partial \underline{\chi}^{+-}(\omega)}{\partial e_{i}^{\beta}} \frac{\partial\left[\underline{\chi}^{+-}(\omega)\right]^{-1}}{\partial e_{j}^{\alpha}}+\underline{\chi}^{+-}(\omega) \frac{\partial^{2}\left[\underline{\chi}^{+-}(\omega)\right]^{-1}}{\partial e_{i}^{\alpha} \partial e_{j}^{\beta}}\right] \operatorname{sgn}(\omega) d \omega .
$$

Assuming that the electron-hole excitations are weakly affected by the infinitesimal rotation of the magnetic moments, the first term on the right-hand side of the preceding equation is found proportional to $\frac{\partial \xi_{i, \pm}^{2}}{\partial e_{i}^{\alpha}}$, i.e., the change of the local spin fluctuation amplitude squared associated with the moment $\mathbf{M}_{i}$ [25] [see Eq. (C8)], which can be safely neglected. Therefore,

$$
\frac{\partial^{2} \mathcal{E}_{ \pm}}{\partial e_{i}^{\alpha} \partial e_{j}^{\beta}} \simeq-\frac{1}{2 \pi} \operatorname{Im} \operatorname{Tr} \int_{-\infty}^{+\infty} \underline{\chi}^{+-}(\omega) \frac{\partial^{2}\left[\underline{\chi}^{+-}(\omega)\right]^{-1}}{\partial e_{i}^{\alpha} \partial e_{j}^{\beta}} \operatorname{sgn}(\omega) d \omega .
$$

Having obtained the general form, we next focus on the particular system analyzed in the main text constituted of two magnetic atoms sitting on a nonmagnetic surface. In this case, the trace in Eq. (C5) becomes

$$
\begin{aligned}
& \operatorname{Tr}\left(\underline{\chi}^{+-} \underline{\chi}_{\alpha \beta}^{+-}\right)=\left[\chi_{11}^{+-} \chi_{\alpha \beta, 11}^{+-}+\chi_{12}^{+-} \chi_{\alpha \beta, 21}^{+-}\right] \\
& +\left[\chi_{21}^{+-} \chi_{\alpha \beta, 12}^{+-}+\chi_{22}^{+-} \chi_{\alpha \beta, 22}^{+-}\right] \text {, }
\end{aligned}
$$

where we have introduced $\chi_{\alpha \beta, i j}^{+-}=\frac{\partial^{2}\left[\underline{\chi}^{+-}(\omega)\right]^{-1}}{\partial e_{i}^{\alpha} \partial e_{j}^{\beta}}$ for conciseness. To evaluate these terms, we make use of the analytical results provided in Eq. (B8) derived via the LLG equation.

Starting from an out-of-plane orientation of the magnetic moments and bearing in mind the relations in Eq. (B5), the diagonal components (i.e., $\alpha \alpha=x x, y y)$ of $\frac{\partial^{2} \mathcal{E}_{ \pm}}{\partial e_{i}^{\alpha} \partial e_{j}^{\beta}}$ are given by

$$
\frac{\partial^{2} \mathcal{E}_{ \pm}}{\partial e_{1}^{\alpha} \partial e_{2}^{\alpha}}=J_{12}^{\alpha \alpha}\left[\frac{\xi_{1, \pm}^{2}}{M_{1}^{2}}+\frac{\xi_{2, \pm}^{2}}{M_{2}^{2}}+\frac{J_{12}^{z z}}{J_{12}^{\alpha \alpha}} \frac{\xi_{12, \pm}^{2}}{M_{1} M_{2}}\right]
$$

where $\xi_{i, \pm}^{2}$ designates the aforementioned square of the local spin fluctuation amplitude of the moment $\mathbf{M}_{i}$ [25],

$$
\xi_{i, \pm}^{2}=\frac{1}{\pi} \operatorname{Im} \int_{-\infty}^{+\infty} \chi_{i i}^{+-}(\omega) \operatorname{sgn}(\omega) d \omega
$$

and the nonlocal contributions $\xi_{i j, \pm}^{2}$ are defined as

$$
\xi_{i j, \pm}^{2}=\frac{1}{2 \pi} \operatorname{Im} \int_{-\infty}^{+\infty}\left[\chi_{i j}^{+-}(\omega)+\chi_{j i}^{+-}(\omega)\right] \operatorname{sgn}(\omega) d \omega .
$$

Note also that the nonlocal spin fluctuation contribution that corrects $J_{12}^{\alpha \alpha}$ (for $\alpha=x, y$ ) in Eq. (C7) is proportional to $J_{12}^{z z}$. In general, these components may differ due to the anisotropy of the system. However, these differences are relevant in the investigated cases when the atoms are far apart [62], where $\xi_{12, \pm}^{2}$ is vanishingly small (as shown in the main text). In this case, Eq. (C7) simplifies to

$$
\frac{\partial^{2} \mathcal{E}_{ \pm}}{\partial e_{1}^{\alpha} \partial e_{2}^{\alpha}}=J_{12}^{\alpha \alpha}\left(\frac{\xi_{1, \pm}^{2}}{M_{1}^{2}}+\frac{\xi_{2, \pm}^{2}}{M_{2}^{2}}+\frac{\xi_{12, \pm}^{2}}{M_{1} M_{2}}\right)
$$


The nondiagonal antisymmetric components can be obtained from Eq. (C5) as well,

$$
\frac{\partial^{2} \mathcal{E}_{ \pm}}{\partial e_{1}^{\alpha} \partial e_{2}^{\beta}}=-J_{12}^{\alpha \beta}\left(\frac{\xi_{1, \pm}^{2}}{M_{1}^{2}}+\frac{\xi_{2, \pm}^{2}}{M_{2}^{2}}+\frac{\xi_{12, \pm}^{2}}{M_{1} M_{2}}\right) .
$$

Finally, we obtain the renormalized exchange interaction tensor components by combining Eqs. (C10) and (C11) into the definition of Eq. (5) and making use of the antisymmetric property $J_{21}^{\alpha \beta}=-J_{12}^{\alpha \beta}$, yielding

$$
\mathcal{J}_{12}^{\alpha \beta}=J_{12}^{\alpha \beta}\left[1-\frac{J_{21}^{\alpha \beta}}{J_{12}^{\alpha \beta}}\left(\frac{\xi_{1, \pm}^{2}}{M_{1}^{2}}+\frac{\xi_{2, \pm}^{2}}{M_{2}^{2}}+\frac{\xi_{12, \pm}^{2}}{M_{1} M_{2}}\right)\right] .
$$

Equation (C12) corresponds to Eq. (7) and shows that diagonal components are decreased by the factor in parentheses, whereas the off-diagonal components are increased by exactly the same relative factor.
[1] T. Moriya and A. Kawabata, Effect of spin fluctuations on itinerant electron ferromagnetism, J. Phys. Soc. Jpn. 34, 639 (1973).

[2] H. B. G. Casimir, On the attraction between two perfectly conducting plates, Proc. K. Ned. Akad. Wet. 51, 793 (1948).

[3] I. Errea, F. Belli, L. Monacelli, A. Sanna, T. Koretsune, T. Tadano, R. Bianco, M. Calandra, R. Arita, F. Mauri, and J. A. Flores-Livas, Quantum crystal structure in the 250-kelvin superconducting lanthanum hydride, Nature (London) 578, 66 (2020).

[4] A. Morello, A. Millán, and L. J. de Jongh, Observation of Zero-Point Quantum Fluctuations of a Single-Molecule Magnet through the Relaxation of its Nuclear Spin Bath, Phys. Rev. Lett. 112, 117202 (2014).

[5] E. Shahmoon and U. Leonhardt, Electronic zero-point fluctuation forces inside circuit components, Sci. Adv. 4, eaaq0842 (2018).

[6] A. A. Varlamov, A. Galda, and A. Glatz, Fluctuation spectroscopy: From Rayleigh-Jeans waves to Abrikosov vortex clusters, Rev. Mod. Phys. 90, 015009 (2018).

[7] T. Moriya and K. Ueda, Antiferromagnetic spin fluctuation and superconductivity, Rep. Prog. Phys. 66, 1299 (2003).

[8] P. A. Lee, N. Nagaosa, and X.-G. Wen, Doping a Mott insulator: Physics of high-temperature superconductivity, Rev. Mod. Phys. 78, 17 (2006).

[9] E. A. Stepanov, L. Peters, I. S. Krivenko, A. I. Lichtenstein, M. I. Katsnelson, and A. N. Rubtsov, Quantum spin fluctuations and evolution of electronic structure in cuprates, npj Quantum Mater. 3, 54 (2018).

[10] P. Dai, Antiferromagnetic order and spin dynamics in iron-based superconductors, Rev. Mod. Phys. 87, 855 (2015).

[11] M. H. Christensen, P. P. Orth, B. M. Andersen, and R. M. Fernandes, Emergent Magnetic Degeneracy in Iron Pnictides Due to the Interplay between Spin-Orbit Coupling and Quantum Fluctuations, Phys. Rev. Lett. 121, 057001 (2018).

[12] M. Brando, D. Belitz, F. M. Grosche, and T. R. Kirkpatrick, Metallic quantum ferromagnets, Rev. Mod. Phys. 88, 025006 (2016).

[13] A. Roldán-Molina, M. J. Santander, A. S. Nunez, and J. Fernández-Rossier, Quantum fluctuations stabilize skyrmion textures, Phys. Rev. B 92, 245436 (2015).

[14] A. L. Wysocki, A. Kutepov, and V. P. Antropov, Strength and scales of itinerant spin fluctuations in $3 d$ paramagnetic metals, Phys. Rev. B 94, 140405(R) (2016).

[15] A. L. Wysocki, V. N. Valmispild, A. Kutepov, S. Sharma, J. K. Dewhurst, E. K. U. Gross, A. I. Lichtenstein, and V. P. Antropov, Spin-density fluctuations and the fluctuation- dissipation theorem in $3 d$ ferromagnetic metals, Phys. Rev. B 96, 184418 (2017).

[16] A. Z. Solontsov and D. Wagner, Spin anharmonicity and zeropoint fluctuations in weak itinerant electron magnets, J. Phys.: Condens. Matter 6, 7395 (1994).

[17] V. P. Antropov and A. Solontsov, The influence of quantum spin fluctuations on magnetic instability, J. Appl. Phys. 109, 07E116 (2011).

[18] P. Gambardella, S. Rusponi, M. Veronese, S. S. Dhesi, C. Grazioli, A. Dallmeyer, I. Cabria, R. Zeller, P. H. Dederichs, K. Kern, C. Carbone, and H. Brune, Giant magnetic anisotropy of single cobalt atoms and nanoparticles, Science 300, 1130 (2003).

[19] F. Meier, L. Zhou, J. Wiebe, and R. Wiesendanger, Revealing magnetic interactions from single-atom magnetization curves, Science 320, 82 (2008).

[20] S. Loth, S. Baumann, C. P. Lutz, D. M. Eigler, and A. J. Heinrich, Bistability in atomic-scale antiferromagnets, Science 335, 196 (2012).

[21] J. Honolka, A. A. Khajetoorians, V. Sessi, T. O. Wehling, S. Stepanow, J.-L. Mi, B. B. Iversen, T. Schlenk, J. Wiebe, N. B. Brookes, A. I. Lichtenstein, P. Hofmann, K. Kern, and R. Wiesendanger, In-Plane Magnetic Anisotropy of Fe Atoms on $\mathrm{Bi}_{2} \mathrm{Se}_{3}(111)$, Phys. Rev. Lett. 108, 256811 (2012).

[22] A. J. Heinrich, J. A. Gupta, C. P. Lutz, and D. M. Eigler, Singleatom spin-flip spectroscopy, Science 306, 466 (2004).

[23] A. A. Khajetoorians, T. Schlenk, B. Schweflinghaus, M. dos Santos Dias, M. Steinbrecher, M. Bouhassoune, S. Lounis, J. Wiebe, and R. Wiesendanger, Spin Excitations of Individual Fe Atoms on Pt(111): Impact of the Site-Dependent Giant Substrate Polarization, Phys. Rev. Lett. 111, 157204 (2013).

[24] Q. Dubout, F. Donati, C. Wäckerlin, F. Calleja, M. Etzkorn, A. Lehnert, L. Claude, P. Gambardella, and H. Brune, Controlling the Spin of Co Atoms on $\mathrm{Pt}(111)$ by Hydrogen Adsorption, Phys. Rev. Lett. 114, 106807 (2015).

[25] J. Ibañez-Azpiroz, M. dos Santos Dias, S. Blügel, and S. Lounis, Zero-point spin-fluctuations of single adatoms, Nano Lett. 16, 4305 (2016).

[26] J. Ibañez-Azpiroz, M. dos Santos Dias, S. Blügel, and S. Lounis, Spin-fluctuation and spin-relaxation effects of single adatoms from first principles, J. Phys.: Condens. Matter 30, 343002 (2018).

[27] L. Zhou, J. Wiebe, S. Lounis, E. Vedmedenko, F. Meier, S. Blügel, P. H. Dederichs, and R. Wiesendanger, Strength and directionality of surface Ruderman-Kittel-Kasuya-Yosida interaction mapped on the atomic scale, Nat. Phys. 6, 187 (2010).

[28] A. A. Khajetoorians, J. Wiebe, B. Chilian, S. Lounis, S. Blügel, and R. Wiesendanger, Atom-by-atom engineering and 
magnetometry of tailored nanomagnets, Nat. Phys. 8, 497 (2012).

[29] A. A. Khajetoorians, J. Wiebe, B. Chilian, and R. Wiesendanger, Realizing all-spin-based logic operations atom by atom, Science 332, 1062 (2011).

[30] J. Hermenau, J. Ibañez-Azpiroz, C. Hübner, A. Sonntag, B. Baxevanis, K. T. Ton, M. Steinbrecher, A. A. Khajetoorians, M. dos Santos Dias, S. Blügel, R. Wiesendanger, S. Lounis, and J. Wiebe, A gateway towards non-collinear spin processing using three-atom magnets with strong substrate coupling, Nat. Commun. 8, 642 (2017).

[31] J. Hermenau, S. Brinker, M. Marciani, M. Steinbrecher, M. dos Santos Dias, R. Wiesendanger, S. Lounis, and J. Wiebe, Stabilizing spin systems via symmetrically tailored RKKY interactions, Nat. Commun. 10, 2565 (2019).

[32] Y. O. Kvashnin, O. Grånäs, I. Di Marco, M. I. Katsnelson, A. I. Lichtenstein, and O. Eriksson, Exchange parameters of strongly correlated materials: Extraction from spin-polarized density functional theory plus dynamical mean-field theory, Phys. Rev. B 91, 125133 (2015).

[33] S. Keshavarz, Y. O. Kvashnin, I. Di Marco, A. Delin, M. I. Katsnelson, A. I. Lichtenstein, and O. Eriksson, Layer-resolved magnetic exchange interactions of surfaces of late $3 d$ elements: Effects of electronic correlations, Phys. Rev. B 92, 165129 (2015).

[34] M. T. Béal-Monod, S.-K. Ma, and D. R. Fredkin, Temperature Dependence of the Spin Susceptibility of a Nearly Ferromagnetic Fermi Liquid, Phys. Rev. Lett. 20, 929 (1968).

[35] S. Lounis, A. T. Costa, R. B. Muniz, and D. L. Mills, Dynamical Magnetic Excitations of Nanostructures from First Principles, Phys. Rev. Lett. 105, 187205 (2010).

[36] S. Lounis, A. T. Costa, R. B. Muniz, and D. L. Mills, Theory of local dynamical magnetic susceptibilities from the Korringa-Kohn-Rostoker Green function method, Phys. Rev. B 83, 035109 (2011).

[37] M. dos Santos Dias, B. Schweflinghaus, S. Blügel, and S. Lounis, Relativistic dynamical spin excitations of magnetic adatoms, Phys. Rev. B 91, 075405 (2015).

[38] B. Rousseau, A. Eiguren, and A. Bergara, Efficient computation of magnon dispersions within time-dependent density functional theory using maximally localized Wannier functions, Phys. Rev. B 85, 054305 (2012).

[39] Physics of Spin in Solids: Materials, Methods and Applications, edited by S. Halilov, NATO Science Series II: Mathematics, Physics and Chemistry (Springer Science + Business Media, Dordrecht, 2006), Vol. 156.

[40] L. Ortenzi, I. I. Mazin, P. Blaha, and L. Boeri, Accounting for spin fluctuations beyond local spin density approximation in the density functional theory, Phys. Rev. B 86, 064437 (2012).

[41] T. Moriya, Developments of the theory of spin fluctuations and spin fluctuation-induced superconductivity, Proc. Jpn. Acad. B 82, 1 (2006).

[42] A. I. Liechtenstein, M. I. Katsnelson, V. P. Antropov, and V. A. Gubanov, Local spin density functional approach to the theory of exchange interactions in ferromagnetic metals and alloys, J. Magn. Magn. Mater. 67, 65 (1987).

[43] L. Udvardi, L. Szunyogh, K. Palotás, and P. Weinberger, Firstprinciples relativistic study of spin waves in thin magnetic films, Phys. Rev. B 68, 104436 (2003).
[44] H. Ebert and S. Mankovsky, Anisotropic exchange coupling in diluted magnetic semiconductors: Ab initio spin-density functional theory, Phys. Rev. B 79, 045209 (2009).

[45] A. Fert, N. Reyren, and V. Cros, Magnetic skyrmions: Advances in physics and potential applications, Nat. Rev. Mater. 2, 17031 (2017).

[46] M. A. Ruderman and C. Kittel, Indirect exchange coupling of nuclear magnetic moments by conduction electrons, Phys. Rev. 96, 99 (1954).

[47] T. Kasuya, A theory of metallic ferro- and antiferromagnetism on Zener's model, Prog. Theor. Phys. 16, 45 (1956).

[48] K. Yosida, Magnetic properties of Cu-Mn alloys, Phys. Rev. 106, 893 (1957).

[49] B. Schweflinghaus, M. dos Santos Dias, A. T. Costa, and S. Lounis, Renormalization of electron self-energies via their interaction with spin excitations: A first-principles investigation, Phys. Rev. B 89, 235439 (2014).

[50] B. Schweflinghaus, M. dos Santos Dias, and S. Lounis, Observing spin excitations in $3 d$ transition-metal adatoms on $\operatorname{Pt}(111)$ with inelastic scanning tunneling spectroscopy: A firstprinciples perspective, Phys. Rev. B 93, 035451 (2016).

[51] F. S. M. Guimarães, M. dos Santos Dias, B. Schweflinghaus, and S. Lounis, Engineering elliptical spin-excitations by complex anisotropy fields in $\mathrm{Fe}$ adatoms and dimers on $\mathrm{Cu}(111)$, Phys. Rev. B 96, 144401 (2017).

[52] N. Papanikolaou, R. Zeller, and P. H. Dederichs, Conceptual improvements of the KKR method, J. Phys.: Condens. Matter 14, 2799 (2002).

[53] D. S. G. Bauer, Development of a relativistic full-potential firstprinciples multiple scattering Green function method applied to complex magnetic textures of nanostructures at surfaces, Ph.D. thesis, Forschungszentrum Jülich, 2014, available at http://publications.rwth-aachen.de/record/229375.

[54] T. L. Gilbert, A phenomenological theory of damping in ferromagnetic materials, IEEE Trans. Magn. 40, 3443 (2004).

[55] J. Ibañez-Azpiroz, M. dos Santos Dias, S. Blügel, and S. Lounis, Longitudinal and transverse spin relaxation times of magnetic single adatoms: An ab initio analysis, Phys. Rev. B 96, 144410 (2017).

[56] S. Holzberger, T. Schuh, S. Blügel, S. Lounis, and W. Wulfhekel, Parity Effect in the Ground State Localization of Antiferromagnetic Chains Coupled to a Ferromagnet, Phys. Rev. Lett. 110, 157206 (2013).

[57] O. M. Sotnikov, V. V. Mazurenko, J. Colbois, F. Mila, M. I. Katsnelson, and E. A. Stepanov, Quantum skyrmions, arXiv:2004.13526.

[58] D. Krause and P. Thörnig, JURECA: Modular supercomputer at Jülich Supercomputing Centre, J. Large-Scale Res. Fac. 4, A62 (2018).

[59] J. Hubbard, Electron correlations in narrow energy bands, Proc. R. Soc. London Ser. A 276, 238 (1963).

[60] N. B. Melnikov and B. I. Reser, Dynamic Spin Fluctuation Theory of Metallic Magnetism (Springer, Berlin, 2018).

[61] G. Giuliani and G. Vignale, Quantum Theory of the Electron Liquid (Cambridge University Press, Cambridge, 2005).

[62] J. Bouaziz, M. dos Santos Dias, A. Ziane, M. Benakki, S. Blügel, and S. Lounis, Chiral magnetism of magnetic adatoms generated by Rashba electrons, New J. Phys. 19, 023010 (2017). 